\title{
Fretting Corrosion and the Reliability of Multicontact Connector Terminals
}

\author{
Jonathan Swingler and John W. McBride
}

\begin{abstract}
The harsh operating environment of the automotive application makes the semi-permanent connector susceptible to intermittent high contact resistance which eventually leads to failure. Fretting corrosion is often the cause of these failures. However, laboratory testing of sample contact materials produce results that do not correlate with commercially tested connectors.

A multicontact (M-C) reliability model is developed to bring together the fundamental studies and studies conducted on commercially available connector terminals. It is based on fundamental studies of the single contact interfaces and applied to commercial multicontact terminals. The model takes into consideration firstly, that a single contact interface may recover to low contact resistance after attaining a high value and secondly, that a terminal consists of more than one contact interface. For the connector to fail, all contact interfaces have to be in the failed state at the same time.
\end{abstract}

Index Terms-Connector reliability, contact resistance, fretting, multiple contacts.

\section{NOMENCLATURE}

a Weibull shape parameter.

$b \quad$ Weibull scale parameter (Life characteristic).

$n \quad$ Number of contact interfaces per terminal.

$R(x, n) \quad$ Reliability function.

$R_{1}(x, n)$ Reliability function for 1 failure and recovery.

$w(x) \quad$ Probability density function of failure.

$w_{M}(x) \quad$ Probability density function of the $M$ th failure.

$W(x) \quad$ Unreliability function or probability of being in failed state.

$W_{M}(x) \quad$ Probability of being in $M$ th failed state.

$x \quad$ Fretting cycle number.

$x_{f} \quad$ Mean cycle number to failure.

$x_{r} \quad$ Mean number of cycles in failed state until recovery.

$\sigma_{f} \quad$ Standard deviation of failure.

$\Gamma(x) \quad$ Gamma function.

$\Omega(x) \quad$ Probability that an interface has failed and recovered.

$\Omega_{M}(x) \quad$ Probability that an interface has failed and recovered $M$ times.

\section{INTRODUCTION}

$\mathbf{T}$ HE ELECTRICAL connector used in both electrical and electronic power systems is often the focus of attention where reliability issues are concerned. When the "no fault

Manuscript received January 11, 2001; revised November 26, 2002. This work was recommended for publication by Associate Editor M. Braunovic upon evaluation of the reviewers' comments.

The authors are with the School of Engineering Science, University of Southampton, Southampton S031 5RR, UK (e-mail: j@ swingler.net).

Digital Object Identifier 10.1109/TCAPT.2002.808007 found" scenario occurs after an inspection of a failed system, it is often attributed to a faulty connection. The connector can fail exhibiting high contact resistance but returns to low resistance due to a self-healing mechanism. Thus no fault is found. Fretting corrosion at the contact interface can cause these failures and is common in the automotive application due to harsh operating environments. A previous study has shown that the operating temperature of the connector is a complex interaction of driving conditions and the external environment [1], [2].

Laboratory fretting tests on contact samples produce results that are not always correlated with data acquired from commercially available connector samples, ref. [3, pp. 341-342]. In tests single contact interfaces have achieved high contact resistance $(0.2 \mathrm{~m} \Omega)$ within a few hundred fretting cycles [4] compared to connector samples where the majority never give a resistance value above $3 \mathrm{~m} \Omega$ for the same test conditions [2]. Work presented here demonstrates that the multicontact nature of connector terminal, combined with the fact that the contact interface can recover after a failure, explains this lack of correlation.

The definition of "multicontact" terminal as used in this study is given in the Appendix-A1 as several independent contacts on a terminal.

\section{SURVEY OF EXPERIMENTAL INVESTIGATIONS}

\section{A. Single Contact Fretting Tests}

Experimental investigations into fretting corrosion of tin and its alloys have been reported by many authors [4]-[6]. These studies investigate contact phenomena involving a single contact interface. Results previously presented by the authors have demonstrated the effect on contact resistance for clean, lubricated, powered and powered lubricated sample types [4]. These samples arranged as a flat and rider were caused to fret with a relative oscillatory movement of $80 \mu \mathrm{m}$ at a frequency of $3 \mathrm{mHz}$. Contact resistance measurements were taken during the fretting cycle. Fig. 1 shows graphs of these four samples types. They are plots of highest and lowest contact resistance per cycle against cycle number. The dark blocked in areas on the graphs indicate the limit of the highest and lowest contact resistance. Cleaned samples fail [attaining $0.2 \Omega$ in Fig. 1(a)] after on average 100 cycles of fretting and recover to low contact resistance. These samples repeatedly fail and recover until around 200 cycles and remain in the failed state thereafter. Lubricated samples [Fig. 1(b)] fail at a later cycle number than clean samples. Failure and recovery cycling occurs for lubricated [Fig. 1(b)] samples across the 1000 cycle tested. 


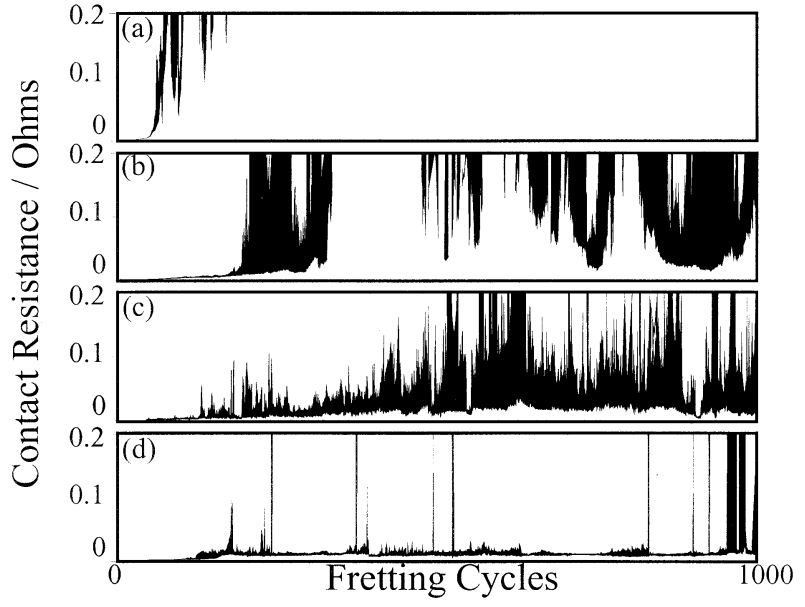

Fig. 1. Fretting test of single contact interfaces: (a) clean, (b) lubricated, (c) powered, and (d) powered lubricated sample types.

Powered [Fig. 1(c)] and powered/lubricated [Fig. 1(d)] samples also showed a failure and recovery cycling process.

Typically, such contact resistance plots are divided into three stages [7], an initial stage of decrease in contact resistance, followed by a contact stable region, followed by an increase in contact resistance. From Fig. 1(a)-(d), a continuous low resistance stage is observed where the contact interface is in a "stable state" under any condition. (Closer inspection of the data also shows the initial decrease in contact resistance which in not seen in Fig. 1.) The final stage, however, not only has an increase in contact resistance, but repeated failure and recovery to low contact resistance. This final stage is denoted as an "unstable state" in this paper.

\section{B. Connector Terminal Fretting Tests}

Fretting tests have also been conducted on commercially available connector terminals [2] which demonstrated a higher level of reliability compared to the single contact interface. These tests were conducted under same conditions as the single contact interface. However, the terminals used have at least two contact interfaces and use a lubricant. Fig. 2 is a plot of contact resistance for twelve terminal types when fretted for 1000 cycles. The average contact resistance values measured is plotted in this graph for each terminal type. The error bars indicate the maximum and minimum values measured during the 1000 cycles. It can be seen that most terminal types have a contact resistance below $3 \mathrm{~m} \Omega$ for the duration of the test. The $\mathrm{M}-\mathrm{C}$ model is used to explain the low contact resistance during this test. (The high average resistance values of terminal type $1 \mathrm{cl}$ and $5 \mathrm{~b}$ is thought to be due to low contact forces and not dealt with here.)

\section{DeVElopment OF THE M-C Model}

The multicontact (M-C) model presented here is developed in three steps. The model is used to evaluate the reliability function of a population of terminals with " $n$ " number of contact interfaces on each terminal. This function demonstrates the influence of multicontact interfaces in commercially available terminals

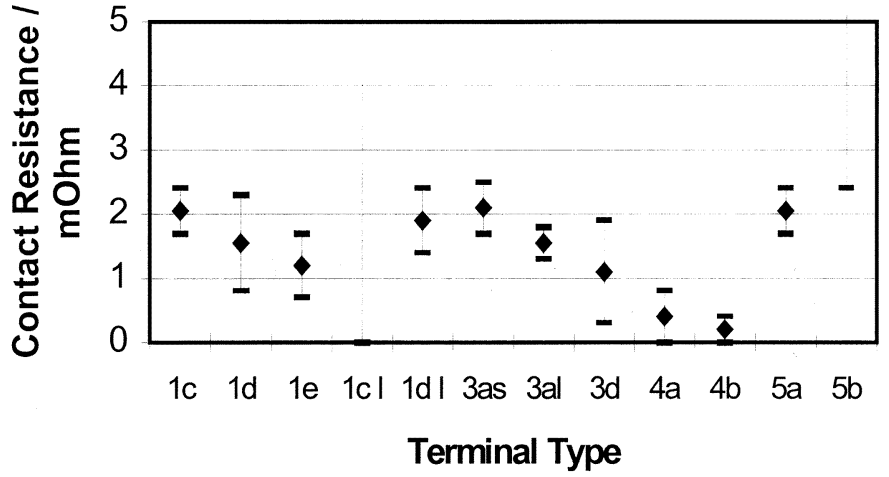

Fig. 2. Fretting tests of terminals

compared to a single interface often studied in fretting tests. In the first step in developing the model, permanent failures are considered for each contact interface. The resultant affect on the reliability function for " $n$ " contacts is shown. Secondly, a failure followed by a recovery is considered and the resultant affect on its reliability function is shown. Finally, many failure and recoveries are considered with the time duration in the failed state assumed to be a fixed value. The affect on the reliability function under this condition is shown.

\section{A. Reliability and the Probability Density Function}

The reliability function $R(x)$ for a single terminal gives the probability that the terminal is not in its failed state by a particular cycle number. Applying this to a population of similar terminals, the number of terminals working at that cycle number can be calculated. The reliability function relates to the unreliability function $W(x)$, otherwise known as the probability of being in the failed state, by

$$
R(x)=1-W(x) .
$$

For a parallel system such as the multicontact terminal, the reliability function is given by the product of the unreliability function for each contact interface [9]

$$
R(x, n)=1-\prod_{i=1}^{n} W_{n}(x)
$$

Assuming the unreliability of each contact interface is the same, (2) becomes

$$
R(x, n)=1-(W(x))^{n} .
$$

The probability of a terminal being in the failed state (unreliability function), $W(x)$, at any particular cycle number is found by integrating the probability density function of failure $w(x)$

$$
W(x)=\int_{0}^{x} w(x) d x .
$$

The probability density function of a terminal is the probability that the terminal will fail at a particular cycle number. A Weibull distribution is used for the probability density function in this analysis. 


\section{B. Data Used in Model}

The model presented here is based on fundamental fretting studies on a single contact interface [4]. Four types of interface have been considered, clean, lubricated, powered, and lubricated/powered interfaces, but only the lubricated interface is described here in detail. Failure is defined as the contact resistance reaching $200 \mathrm{~m} \Omega$.

\section{The Model And Results}

\section{A. Reliability Function for Permanent Failure}

A permanent failure of the contact interface is considered in this first step, i.e., once the contact interface reaches a high contact resistance it is considered to remain high. The Weibull probability density function of failure for a population of terminals for this condition is given [9]

$$
w(x)=\frac{a}{b^{a}} x^{a-1} \exp \left(-\left(\frac{x}{b}\right)^{a}\right)
$$

where " $a$ " is a shape parameter of the distribution and " $b$ " is a scale parameter (life characteristic of contact interface). The $a$ and $b$ Weibull parameters were determined from experimental data expressed for a Gaussian distribution in ref. [4], as the mean cycle number to failure, $x_{f}$, and standard divination, $\sigma_{f}$. The following relationships were used to determine the $a$ and $b$ parameters [10]

$$
\begin{aligned}
& x_{f}=b \Gamma\left(1+a^{-1}\right) \\
& \sigma_{f}=b \sqrt{\Gamma\left(1+2 a^{-1}\right)-\Gamma^{2}\left(1+a^{-1}\right)} .
\end{aligned}
$$

The probability of failure, $W(x)$, between cycle number 0 and $x$ is calculated using data in Table I, and (4) and (5). The reliability function $R(x, n)$ of a terminal with $n$ contact interfaces can then be found from the probability of failure using (3).

The reliability function for a population of terminals with lubricated interfaces are plotted in Fig. 3 for 1, 2, 4, and 8 contacts per terminal. Equation (5), the failure probability density function $w(x)$, is also plotted in Fig. 3 for $n=1$. This peaks at 390 cycles indicating the cycle number which has the highest number of terminals failing.

It can be seen from Fig. 3 that increasing the number of contacts, $n$, increases terminal reliability. The reliability curves moves to the right, so more fretting cycles are required to achieve $50 \%$ failure of the population [where $R(x, n)=0.5$ ]. Additionally, with an increased $n$, at $R(x, n)=0.5$ the failure rate is also increased. It should be noted that this extension to life is not by any great order. Table II shows the percentage extension of cycles from this data for when $50 \%$ of the population has failed.

This analysis assumes that a contact interface does not recover once failed.

\section{B. Reliability Function for One Failure Followed by a Recovery}

The experimental data shows that a permanent failure does not occur and that a recovery to low contact resistance takes place (see Fig. 1). This is accounted for in the M-C model by

\begin{tabular}{|c|c|c|c|c|}
\hline Interface & $x_{f}$ & $\sigma_{\mathrm{f}}$ & $\begin{array}{l}\text { a } \\
\text { (shape) }\end{array}$ & $\begin{array}{l}\mathrm{b} \\
\text { (scale) }\end{array}$ \\
\hline Clean & 100 & 76 & 1.4 & 120 \\
\hline Lube & 350 & 130 & 3.0 & 390 \\
\hline Pow & 500 & 300 & 1.7 & 550 \\
\hline Pow/Lube & 500 & 200 & 2.7 & 570 \\
\hline
\end{tabular}

TABLE I

FAILURE PARAMETERS From EXPERIMENTAL DATA [4]

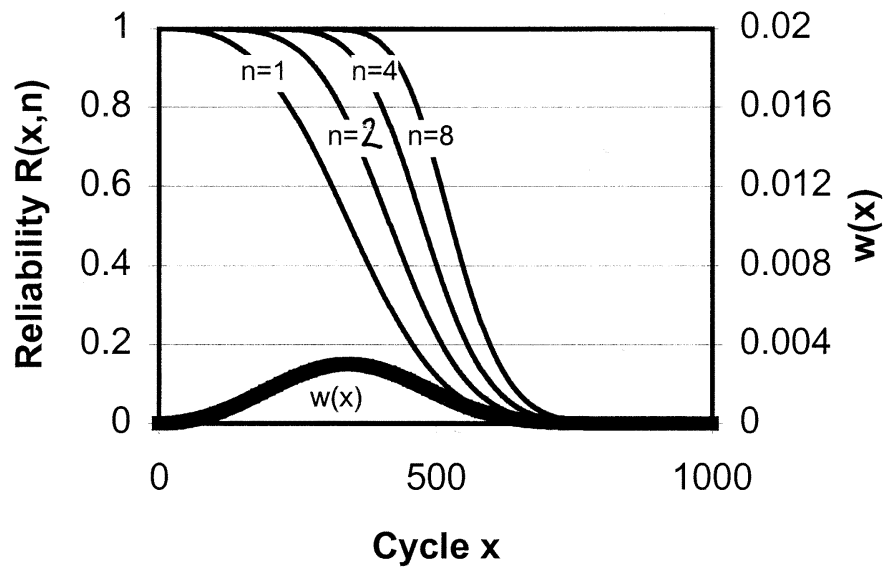

Fig. 3. Reliability function for " $n$ " lubricated contacts and the failure probability distribution function for one contact interface.

TABLE II

INCREASE IN AVERAGE NUMBER OF CYCLES BEFORE FAILURE

\begin{tabular}{c|c}
\hline $\mathrm{n}$ & $\%$ increase \\
\hline 1 & 0 \\
2 & 6.9 \\
4 & 22.3 \\
8 & 35.4 \\
\hline
\end{tabular}

considering that the contact recovers after $x_{r}$ cycles after failure. The probability of a contact interface being in its failed state at cycle $x$ is the probability that the contact will fail between cycle $x-x_{r}$ and $x$. This is calculated by integrating the probability density function between $x-x_{r}$ and $x$, as in (6). Considering a population of terminals with one contact interface and the failed state to last $x_{r}$ cycles, the number of terminals in the failed state will be those that had failed at cycle $x$ and $(x-1)$ and $(x-2)$ .. and $\left(x-x_{r}\right)$. $W_{1}(x)$ for a terminal is the probability that the contact interface is in the failed state at cycle $x$ after it first 


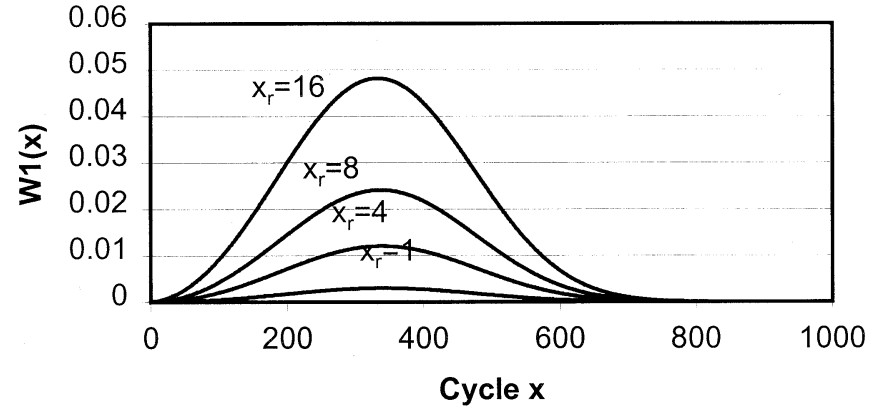

Fig. 4. Probability of the contact interface being in its failed state at particular cycle number.

TABLE III

RECOVERY PARAMETERS FROM EXPERIMENTAL DATA [4]

\begin{tabular}{l|l}
\hline Interface & $\mathrm{x}_{\mathrm{r}}$ \\
\hline Clean & 7.1 \\
\hline Lube & 13.5 \\
\hline Pow & 5.2 \\
\hline Pow/Lube & 4.5 \\
\hline
\end{tabular}

fails and before the first recovery (suffix 1 denotes first failure and recovery)

$$
\begin{aligned}
W_{1}(x) & =\int_{x-x_{r}}^{x} w_{1}(x) d x \\
& =\int_{x-x_{r}}^{x} \frac{a}{b^{a}} x^{a-1} \exp \left(-\left(\frac{x}{b}\right)^{a}\right) d x \\
& =\exp \left(-\left(\frac{x-x_{r}}{b}\right)^{a}\right)-\exp \left(-\left(\frac{x}{b}\right)^{a}\right) .
\end{aligned}
$$

This is plotted in Fig. 4 for different $x_{r}$ for the lubricated case where average failure is at 390 cycles for $n=1$. It can be seen that the longer the contact interface is in the failed state the higher the failure probability for any particular cycle number.

A value for the $x_{r}$ parameter has been found from experimental data for the four sample types. The mean values are given in Table III for the fretting experiments conducted [4]. The lubricated case of $x_{r}=13.5$ is considered in this analysis.

The reliability function for one failure and recovery of the terminal with $n$ contact interfaces is found by

$$
R_{1}(x, n)=1-\left(W_{1}(x)\right)^{n} .
$$

The function is plotted in Fig. 5 for the Lubricated terminal system where $x_{r}=13.5$. Two types of terminals are plotted with $n=1$ and $n=2$.

Considering a terminal of $n=1$ at cycle number 390, the terminal will have a probability of $\sim 0.96$ that it is in the working state (see Fig. 5) or a probability of $\sim 0.04$ that it is in the failed state. It should be noted that the terminal may have already failed and recovered by cycle number 390 and currently be in its

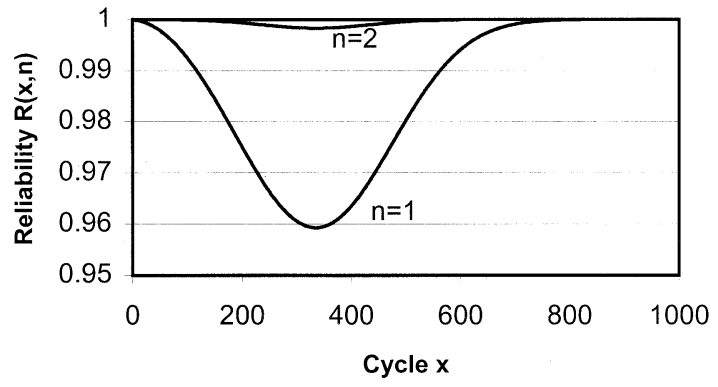

Fig. 5. Terminal reliability for $n=1$ and 2 contacts.

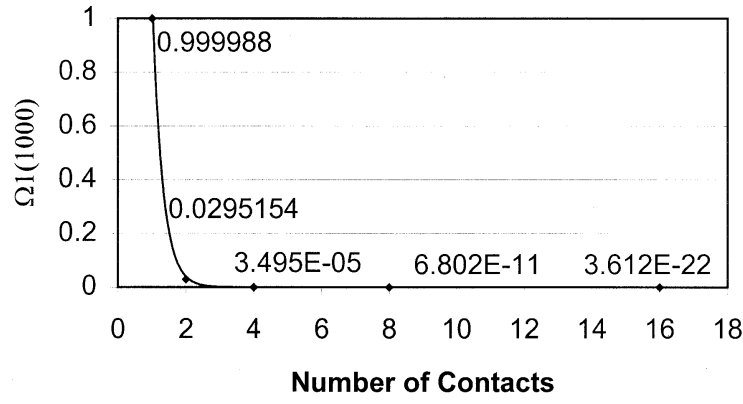

Fig. 6. Probability that the terminal has failed between 0-1000 cycles.

working state. At cycle 1000 a terminal has a very high probability that it is in the working state $(\sim 1)$. However, by cycle 1000 the single contact terminal has probably failed and recovered. The probability that a terminal has failed and recovered, $\Omega_{1}(x)$, can be calculated by integrating the normalized probability $W_{1}(x)$ function between 0 and 1000 cycles giving $\sim 1$, see the Appendix-A2.

Considering a population of similar terminals with $n=1$, at 390 cycles, it would be expected that just over $4 \%$ are in the failed state. For $n=2$, less then $0.5 \%$ are expected in the failed state. By increasing the number of contacts, $n$, in the terminal and including the fact that the contact recovers, increases the reliability significantly.

For multicontact terminal there is a much higher probability of not failing at all. This can be calculated by integrating the normalized $W_{1}(x)^{n}$ function between 0 and 1000 cycles giving $\Omega_{1}(x)$ for different $n$. See the Appendix-A2. This is plotted against the number of contacts, $n$, in Fig. 6.

This analysis is for one failure and recovery. However a contact interface will fail and recover many times.

\section{Reliability Function for Many Failures and Recoveries}

For the contact interface to undergo a second failure, it must have encountered a first failure and recovery. Considering a single contact interface terminal, the probability that the terminal has had one failure and recovery at cycle number $x$ is given by integrating the probability density function of failure (suffix 1 denotes first failure and recovery) as in

$$
\Omega_{1}(x)=\int_{0}^{x} w_{1}(x) d x .
$$

Therefore, the probability of a terminal being in its second failed state is given by (9). This is the product of the probability that the terminal has had one failure and recovery, $\Omega_{1}$, and the 
probability of the second failure. The probability of the second failure is calculated in a similar manner to the first failure as in (6) accept the mean time to failure is now assumed to be $2 x_{f}=2 \times 390$ cycles. This modifies the $a$ and $b$ parameters as shown in

$$
\begin{aligned}
W_{2}(x)= & \Omega_{1}(x) \frac{1}{2} \\
& \cdot\left(\exp \left(-\left(\frac{x / 2-x_{r}}{b}\right)^{2 a}\right)-\exp \left(-\left(\frac{x}{2 b}\right)^{2 a}\right)\right) .
\end{aligned}
$$

Generalizing, before the probability of a terminal being in its $M$ th failed state can be found, the probability of it having failed $M-1$ times has to be found and this is given by $\Omega_{M-1}$. The probability of a terminal at cycle number $x$ which have failed $M-1$ times is given by

$$
\Omega_{M-1}(x)=\prod_{m=1}^{M-1} \int_{0}^{x} w_{m}(x) d x .
$$

Therefore, the probability of a terminal being in its $M$ th failed state is given by (11), the probability that the terminal has had $M-1$ failures and recoveries $\left(\Omega_{M-1}\right)$ and the probability of the $M$ th failure

$$
\begin{aligned}
& W_{M}(x)=\Omega_{M-1}(x) \frac{1}{M} \\
& \quad\left(\exp \left(-\left(\frac{x / M-x_{r}}{b}\right)^{M a}\right)-\exp \left(-\left(\frac{x}{M b}\right)^{M a}\right)\right) .
\end{aligned}
$$

By defining $\Omega_{0}=1$, the reliability function for a total of $\mu$ failures and recoveries and $n$ contact interfaces is given by

$$
R_{\mu}(x, n)=1-\left(\sum_{M=1}^{\mu} W_{M}(x)\right)^{n} .
$$

Equation (12) gives values as plotted as in Fig. 7. This assumes the contact fails and recovers every 390 cycles, where $\mu=5$ as a first approximation and $n=1$.

At any one point in time after 390 cycles a terminal with one contact interface will have a reliability between $\sim 0.96-0.97$. A population of terminals will have $\sim 96-97 \%$ in the working state or $\sim 3-4 \%$ in the failed state. Increasing the number of contacts significantly improves the reliability function. Table IV shows the minimum value for $R(x, n)$ calculated and the percentages of terminals in the failed state for different number of contacts.

\section{DISCUSSION}

It can be seen from the contact resistance measurements in Fig. 1 that this data can be divided into a stable and an unstable state. The stable state occurs at the beginning of the fretting test and is characterized by low resistance. The unstable state is characterized by contact resistance cycling between high and low values, where the interface is in a failure/recovery state.

During the fretting action, high contact resistance is achieved as resistive debris is built up at the ends of the wear track. The recovery to low resistance is achieved by two possible mechanisms. The resistive material can be either 1) dispersed by lubricants on the surface or 2) by electrical breakdown (or fritting). A more detail discussion of these can be found in ref. [8].

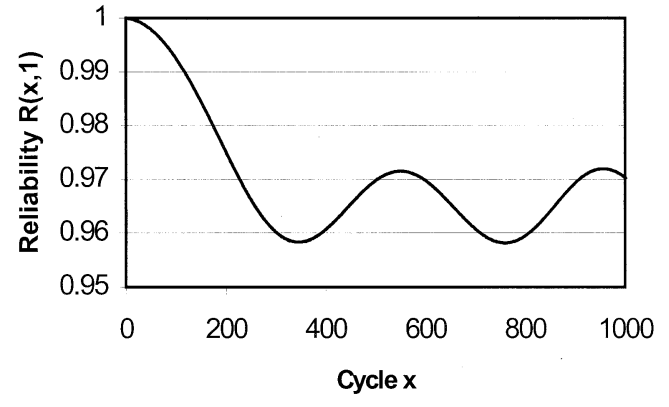

Fig. 7. Reliability of the connector terminal for $n=1$ contact.

TABLE IV

RELATING\% OF CONNECTOR IN FAILURE STATE TO THE NUMBER OF CONTACTS

\begin{tabular}{l|l|l}
\hline $\mathrm{n}$ & Min & Persentage of Terminals in \\
& $\mathrm{R}(\mathrm{x}, \mathrm{n})$ & Failed State \\
\hline 1 & 95.8 & $3-4 \%$ \\
2 & 99.9 & $0.089-0.118 \%$ \\
4 & 100 & $0.105-0.140 \times 10^{-3} \%$ \\
8 & 100 & $2.04-2.72 \quad \times 10^{-10} \%$ \\
\hline
\end{tabular}

\section{A. Stable State}

Fig. 3 and Table II illustrates the effect the number of contact interfaces has on the reliability of the terminal, assuming permanent failure occurs. By increasing the number of contacts per terminal, extends the stable state for the terminal before the permanent failure. This extending feature also applies to when recovery is a possibility, so that the average number of cycles to the first failure of a contact interface is extended.

\section{B. Fail/Recovery State}

Due to the nature of fretting corrosion, a contact interface is not likely to remain in the failed state but a recovery to low resistance is probable. Fig. 7 and Table IV illustrate the effect recovery has on the reliability function. This suggests no more than $4 \%$ of a population of terminals with 1 contact interface being in a failed state at any particular cycle number. Increasing the number of contact interfaces reduces this percentage so that the reliability function is close to $R(x, n)=1$ for more an 4 contacts per terminal.

Commercially available connectors typically have 2 or 4 contact interfaces per terminal. From the data in Table IV, for the 2 contact case $\sim 1$ in 1000 terminals may be in the failed state at any one time. For the 4 contact case this is $\sim 1$ in 1000000 terminals which may be in the failed state at any one time.

\section{Independent Contacts}

Pitney identifies three issues which may effect the correct evaluation of performance using a probabilistic approach, such as used here [11]: 
1) The Normal Force;

2) a general issue of the contacts being physically and electrically connected;

3) cleaning and recovery of the contacts interface.

This third issue is addressed in the M-C model and demonstrates its advantages for improved reliability.

However the other two issues are difficult to address. The $\mathrm{M}-\mathrm{C}$ model assumes constant and same normal forces for each contact interface. It should be noted that using the M-C model of the performance of terminals is assuming that the multicontacts are independent of each other. This is not true in a commercially available terminal. For example let us consider a two contact terminal with contacts made on opposite sides of a pin by two cantilever beams. If the pin is misaligned with respect to the cantilever beams, under fretting conditions, one contact may see an increase in normal force whereas the other may see a decrease in normal force as the pin moves relative to the beams. The two contact interfaces see an equal and opposite condition and cannot be said to be independent.

\section{CONCLUSION}

Laboratory Fretting tests have consisted of a single contact interface and have been useful as a comparative test of materials. With such tests, high contact resistance has been measured after a few hundred, or a few thousand, cycles depending upon test parameters. However, tests upon commercially available connector terminals have not exhibited high contact resistance until much later in fretting tests, in some case no increases have been observed at all.

The multicontact (M-C) model has been presented to explain the lack of correlation between fundamental tests and terminal tests. Two features are considered.

1)A single contact interfaces undergoing fretting corrosion enters an unstable state of failure and recovery.

2)In a multicontact terminal all contact interfaces must be in the failed state before the terminal fails.

The M-C model is evaluated for $1,2,4$, and 8 contact interfaces per terminal in terms of the reliability function. The data used in the single contact interface case is taken from experimental data and extrapolated to the multicontact case. The model considers all contact interfaces to be in parallel.

It was found that there is an exponential improvement to the reliability function with increasing number of contact interfaces.

\section{APPENDIX}

\section{A1. Definition of "Multicontact"}

Asperities and Clusters: The surface of a terminal pin has a roughness associated with it. If two surfaces are considered on a microscopic level, one surface as flat with no roughness and the second surface with a roughness, then when these surfaces are brought together they touch at the peaks of the rough surface. These peaks initially elastically and then plastically deform to support the normal (contact) force. These touching deformed peaks are often called asperities. Several asperities can be formed depending upon the surface properties and normal force applied. These asperities are also known to form in clusters. These two touching surfaces consisting of clusters of asperities are said to form a contact in this study.

Independent Contacts and Multicontacts: As defined in this study of connector terminals, contact number one is said to be independent from a second contact if:

1) any wear track caused by contact one does not overlap a wear track cause by contact two;

2) the normal (contact) force holding the surfaces of contact one together is supplied separately from the force holding contact two together.

It should be noted that for "ideal" independent contacts, external influences on contact one should be different to those on contact two. However, in this definition this is not the case. External influences to contact one, such as the electrical power source, external temperature, vibration, humidity and atmospheric contaminants, may be the same influences upon contact two.

A multicontact terminal is defined as several independent contacts.

\section{A2. Probability That the Terminal Has Failed}

Considering a terminal of $n$ contact interfaces which failures and recovers for the first time has a probability of failure being in the failed state as $W_{1}(x)$ [see (6)]. If $x_{r}=1$ cycle then

$$
W_{1}(x)=\int_{x-1}^{x} w_{1}(x) d x=w_{1}(x) .
$$

The probability that the terminal has failed between cycle 0 and $x=1000$ is

$$
\Omega_{1}(1000)=\int_{0}^{1000} W_{1}(x)^{n} d x \sim 1 .
$$

This is using data for the lubricated case with $n=1$. Integrating this between 0 to $\infty$ gives 1 .

However for the lubricated contact it remains in the failed state for $x_{r}=13.5$ cycles giving a $W_{1}(x)$ function which is 13.5 times higher for each $x$ than in (A1) When integrating this, the probability will be consider more than once each cycle and has to be normalized. Therefore, the probability that a terminal has failed over the range of $0-1000$ cycles is given by

$$
\Omega_{1}(1000)=\frac{1}{x_{r}} \int_{0}^{1000} W_{1}(x)^{n} d x .
$$

Note

$$
\frac{1}{x_{r}} \int_{0}^{1000} W_{1}(x)^{1} d x=\int_{0}^{1000} w_{1}(x) d x .
$$

\section{REFERENCES}

[1] J. Swingler, J. W. McBride, and C. Maul, "Degradation of road tested automotive connectors," IEEE Trans. Comp. Packag. Technol., vol. 23, pp. 157-164, Mar. 2000.

[2] J. Swingler and J. W. McBride, "The synergistic relationship of stresses in the automotive connector," in Proc. 19th Int. Conf. Electric Contact Phenom., Nuremberg, Germany, 1998, pp. 141-145.

[3] M. Antler, Tribology of Electronic Connectors in Electrical Contacts: Principles and Application, P. G. Slade, Ed., 1999. 
[4] J. Swingler, "The automotive connector," in Proc. ImechE Trans. Part $D, 2000$, pp. 615-623.

[5] A. Rudolphi et al., "Fretting testing of electrical contacts at small displacement amplitudes-Experience from a BriteEuram Project," in Proc. Int. Conf. Electrical Contacts, 2002, pp. 471-476.

[6] M. Antler, Fretting of Electrical Contacts: Materials Evaluation Under Fretting Conditions, S. R. Brown, Ed. New York: ASTM, 1982, pp. 68-85.

[7] L. Boyer and L. Tristani, "A model for the contact resistance evolution during a fretting test," in Proc. Int. Conf. Electrical Contacts, 2000, pp. 457-463.

[8] J. Swingler, "The degradation of electrical contacts under low frequency fretting condition," Ph.D. dissertation, Loughborough University, Loughborough, UK, 1994.

[9] D. Kececioglu, Reliability Engineering Handbook. Englewood Cliffs, NJ: Prentice Hall, 1991, vol. I and II.

[10] Microsoft Excel, Tech. Rep., 2002.

[11] K. E. Pitney, Ney Contact Manual: Ney, 1973, pp. 156-160.

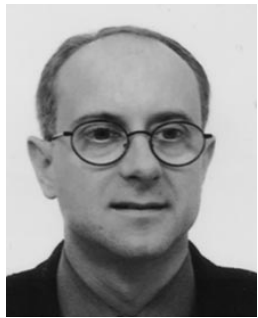

Jonathan Swingler received the M.S. degree in physics and chemistry from the University of Keele, U.K., in 1990 and the Ph.D. degree from Loughborough University, U.K., In 1994. His thesis was on the "Degradation of Electrical Contacts Under Low Frequency Fretting Conditions."

Since then, he has been pursuing research in the area of electrical contact science and technology at the University of Southampton, U.K., and is currently a Senior Research Fellow in the Electromechanical Research Group.

Dr. Swingler is a Chartered Physicist in the U.K.

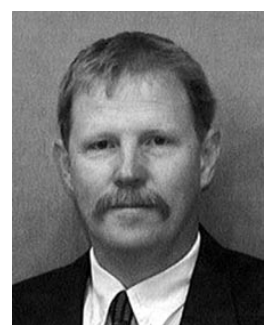

John W. McBride received the M.S. degree in aeronautical engineering from the University of Southampton, Southampton, U.K., in 1978 and the $\mathrm{Ph} . \mathrm{D}$. degree in electrical contact phenomena from Plymouth University, Plymouth, UK, in 1986.

From 1985 to 1987, he lectured in the Mechanical Engineering Department, Plymouth University, and since 1987, has been a Lecturer, Senior Lecturer, and a Reader in instrumentation and measurement in the School of Engineering Science and the Electrical Engineering Department, University of Southampton. Since 2001, he has been Professor of electromechanical engineering in the School of Engineering Science. He is Chair of the Electromechanical Research Group, and Deputy Head of the School. His main research interests include electrical contacts, metrology, and instrumentation.

Dr. McBride is an Associate Editor of the IEEE TRAnsactions on Components and Packaging Technologies, a Chartered Engineer, and Chair of the Electro-Mechanical Research Group, and Vice Chair of IEE professional group S3 Electron Physics, Plasmas, and applications. He is a member of the Organizing Committee, IEEE Holm Conference on Electrical Contacts. 